\title{
Occult Breast Carcinoma
}

National Cancer Institute

\section{Source}

National Cancer Institute. Occult Breast Carcinoma. NCI Thesaurus. Code C137674.

Breast carcinoma presenting with isolated axillary lymphadenopathy, without clinical or mammographic evidence of breast tumor. 